ISSN: $1130-3743$

\title{
HACIA UNA TEORÍA SOBRE EL RENDIMIENTO ACADÉMICO EN ENSEÑANZA PRIMARIA A PARTIR DE LA INVESTIGACIÓN EMPÍRICA: DATOS PRELIMINARES
}

\author{
Towards a theory of academic performance in primary \\ education based on empirical research: preliminary data
}

Vers une théorie du rendement académique dans

l'enseignement primaire à partir de la recherche empirique: données préliminaires

Santiago NieTo MarTín

Universidad de Salamanca. Facultad de Educación. Departamento de Didáctica, Organización y Métodos de Investigación en Educación. Paseo de Canalejas, 169. 37008 Salamanca. Correo-e: snietom@usal.es

Fecha de recepción: enero de 2008

Fecha de aceptación definitiva: abril de 2008

BIBLID [(1130-3743) 20, 2008, 249-274]

RESUMEN

La investigación educativa sobre el rendimiento académico ha formado parte de una constante de indagación y conocimiento sobre el tema durante los últimos treinta años en nuestro país. Sin embargo, el gran volumen de trabajo acumulado no parece haber contribuido suficientemente a la creación de una teoría consistente sobre el rendimiento académico, y, mucho menos, haber seguido la estela de exigencia que comporta la creación de teorías científicas sobre la cuestión. Se produce la constatación de gran cantidad de tendencias, pero no se establecen conocimientos de mayor alcance dadas las limitaciones de los diseños metodológicos bajo los que se han llevado a cabo la mayoría de las investigaciones. 
Ante esta situación, proponemos la consecución de las denominadas teorías de alcance intermedio con el fin de desarrollar un esquema conceptual progresivo más general que promueva la consolidación de grupos de teorías especiales, de las cuales derivar hipótesis que se puedan investigar empíricamente con mayor profundidad.

Palabras clave: teoría científica, rendimiento académico, enseñanza primaria, investigación empírica, validez externa, teorías de alcance intermedio.

\section{SUMMARY}

Educational research into academic performance has formed part of a constant subject of study in the last thirty years in Spain. However, the large volume of work that has been accumulated does not seem to have contributed sufficiently to the creation of a coherent theory of academic performance, and much less to have followed the path demanded of scientific theories on the matter. A large amount of trends are confirmed, but knowledge of a broader scope has not been established given the limitations of the methodological designs under which most of the research has been carried out.

Faced with this situation, we propose to attain so-called middle range theories in order to develop a more general progressive conceptual scheme to promote the consolidation of groups of special theories from which to derive hypotheses that can be investigated empirically in greater depth.

Key words: scientific theory, academic performance, primary education, empirical research, external validity, middle range theories.

SOMMAIRE

Depuis les années 70, le rendement académique a toujours été objet de recherche dans notre pays. Or, les nombreux travaux existant à ce propos ne semblent pas avoir contribué suffisamment au développement d'une théorie consistante répondant aux exigences propres aux théories scientifiques sur la matière. Étant donné les limitations des développements méthodologiques de la plupart des recherches menées, un grand nombre de tendances ont été confirmées mais des connaissances d'une plus grande portée n'ont pas pu être établies.

Dans ce travail, on propose donc la consécution des dénommées théories à moyenne portée dans le but de développer un schéma conceptuel progressif plus général promouvant la consolidation de groupes de théorie spéciales à partir desquels on établira des hypothèses permettant une recherche empirique plus profonde.

Mots clef: théorie scientifique, rendement académique, enseignement primaire, recherche empirique, validité externe, théories à portée moyenne. 
HACIA UNA TEORÍA SOBRE EL RENDIMIENTO ACADÉMICO EN ENSEÑANZA PRIMARIA A PARTIR DE LA INVESTIGACIÓN EMPÍRICA: DATOS PRELIMINARES

\section{INTRODUCCIÓN}

Ya hace medio siglo, Robert Merton, en su Teoría y estructura social, manifestaba que, como muchas palabras excesivamente usadas, la palabra teoría amenazaba quedar vacía de sentido. De igual manera podemos manifestarnos en la época presente; la misma diversidad de "cosas" a que se aplica, desde pequeñas hipótesis de trabajo, pasando por especulaciones generales pero vagas y desordenadas, hasta los sistemas axiomáticos del pensamiento, el empleo de la palabra, con frecuencia, oscurece el entendimiento en vez de aclararlo.

En términos generales, podemos decir que una teoría se refiere a grupos de proposiciones lógicamente interconectados, de los que pueden derivarse uniformidades empíricas, siendo, a su vez, consecuencia de estas últimas. Consideramos, pues, la teoría, como un sistema lógico compuesto de evidencias, axiomas y postulados, que tienen como objetivo declarar bajo qué condiciones se desarrollarán ciertos supuestos, tomando como contexto una explicación del medio idóneo para que se desarrollen las predicciones. A raíz de éstas, se pueden especular, deducir y/o postular mediante ciertas reglas o razonamientos, otros posibles hechos.

Así pues, podemos hablar de teoría científica como un sistema abstracto hipotético-deductivo que constituye una explicación o descripción científica de un conjunto relacionado de observaciones y experimentos; está basada en hipótesis o supuestos verificados por grupos de científicos. En general, contiene varias leyes científicas verificadas, y, en ocasiones, deducibles de la propia teoría.

Ahora bien, en el ámbito científico pedagógico es muy importante resaltar que también se llama teoría a un modelo para el entendimiento de un conjunto de hechos empíricos; y ésta es una perspectiva muy interesante a tener en cuenta en la revisión empírica que pretendemos llevar a cabo. Haciendo uso de los conocimientos que nos proporciona la física, resulta de interés recordar a Stehen Hawking en su obra Breve historia del tiempo, cuando manifiesta que "una teoría es buena si satisface dos requerimientos: debe describir con precisión una extensa clase de observaciones sobre la base de un modelo que contenga sólo unos cuantos elementos arbitrarios, y debe realizar predicciones concretas acerca de los resultados de futuras observaciones"; para después afirmar que

cualquier teoría física es siempre provisional, en el sentido que es sólo una hipótesis; nunca puede ser probada. No importa cuántas veces los resultados de los experimentos concuerdan con alguna teoría, nunca se puede estar seguro de que la próxima vez el resultado no la vaya a contradecir. Por otro lado, se puede refutar una teoría con encontrar sólo una observación que esté en desacuerdo con las predicciones de la misma.

\section{Modelos TEÓRICOS, TIPOS DE TEORÍA E INVESTIGACIÓN CIENTÍfICA}

Construimos, pues, teorías, para explicar, predecir y dominar diferentes fenómenos de la realidad; incluso, la teoría, a veces, es observada como un modelo de 
la propia realidad, como un proceso de generalizaciones acerca de observaciones, que, en todo caso, deben ser verificables.

En cuanto al mundo de las ideas, existen dos categorías que pueden desembocar en teorías; si una suposición no es respaldada, se la conoce como una conjetura; ahora bien, si es respaldada, entonces hablamos de hipótesis, aunque es cierto que muchas hipótesis, al resultar falsas, no evolucionan. Las teorías pueden llegar a ser aceptadas si son capaces de realizar predicciones correctas, y suposiciones más simples tienden a ser aceptadas preferentemente sobre aquellas que son más complejas, constituyendo parte del método científico tanto el proceso de aceptar teorías como el de extender las existentes.

En el plano académico y científico, una teoría llega a constituir un paradigma establecido que explica gran parte o la totalidad de los datos con que se cuenta, ofreciendo predicciones verificables y válidas; en ese caso, una teoría nunca puede ser probada como verdadera porque nunca podemos asumir que sabemos todo lo que hay al respecto.

Las teorías comienzan con observaciones empíricas que generan interrogaciones cuando no simple curiosidad, o bien, la necesidad de saber el porqué de un fenómeno o suceso, lo que conlleva asumir una vía de carácter teorético/científica, que, en la práctica, constituye una combinación de hipótesis que han de orientar la investigación. Como sabemos, en algunos casos, cuando las teorías son ampliamente aceptadas, se pueda hablar de leyes.

Desde el punto de vista de la ciencia, solamente podemos hablar de teoría cuando el conjunto de descripciones tienen una sólida base empírica firme, es decir:

a) es consistente con la teoría preexistente, siempre y cuando esta última haya sido verificada;

b) es sostenida por muchas líneas de evidencia;

c) ha sobrevivido a muchas pruebas críticas que la podrían haber falsificado;

d) hace predicciones que, incluso, podrían ser utilizadas para falsificarla;

e) de entre la gran variedad de explicaciones alternativas sobre los mismos datos, es o resulta la mejor explicación conocida.

La relación entre teoría e investigación científica es clara y manifiesta, puesto que el desarrollo de teorías científicas no sólo es una característica de la lógica, sino también de la racionalidad de la investigación científica, dado que la ciencia muestra su interés por reunir observaciones, desarrollar explicaciones por asociaciones y construir teorías.

Baker $(1997,45)$ pone de relieve que «una teoría es una explicación propuesta para dar dirección a sucesos coordinados o interrelacionados", significando con ello que las teorías son argumentos lógicos que se utilizan para probar las relaciones y supuestos en que se sustenta contrastándolos con la evidencia empírica.

Relevante es la opinión de Kerlinger $(1985,6)$, cuando define la teoría científica como «un conjunto de construcciones hipotéticas (conceptos), definiciones y 
HACIA UNA TEORÍA SOBRE EL RENDIMIENTO ACADÉMICO EN ENSEÑANZA PRIMARIA A PARTIR DE LA INVESTIGACIÓN EMPÍRICA: DATOS PRELIMINARES

proposiciones relacionadas entre sí, que ofrecen un punto de vista sistemático de los fenómenos, al especificar las relaciones existentes entre las variables, con objeto de explicar y predecir los fenómenos".

Yurén (1978) afirma que no existe ciencia si no existe teoría científica, lo que supone admitir que una investigación adquiere el estatus de ciencia siempre que haya sido capaz de construir teorías, de tal modo que si se presentan de forma aislada, tanto los problemas como las hipótesis, no constituyen ciencia.

Las teorías constituyen el verdadero motor de la ciencia, lo que posibilita a los científicos organizar y entender las observaciones, pudiendo permitir o provocar futuras observaciones, existiendo criterios esenciales (lógicos y empíricos) que podemos utilizar para distinguir entre lo que realmente es una teoría científica de lo que no lo es, o bien, tiene mera apariencia de científico. Entre los criterios lógicos más utilizados destacan la parsimonia (usando ideas más simples que no postulen cosas innecesarias), la consistencia lógica, que sea falsable lógicamente y que esté claramente limitada por condicionamientos explícitamente definidos (quedando claro si los datos concretos son relevantes para la verificación o falsación). No menos importantes son los criterios empíricos, entre los que destacan el que sean empiricamente contrastables, lleven a predicciones o retrodicciones que estén verificadas o contrastadas, que impliquen resultados reproducibles y que provean criterios para interpretar los datos como hechos relevantes o irrelevantes.

Una teoría puede y debe cumplir toda una serie de criterios sociológicos, históricos e, incluso, legales, que resumimos en los siguientes términos:

- resolver problemas conocidos, o bien, paradojas o anomalías sin resolver;

- plantear nuevos problemas sobre los que los científicos puedan trabajar;

- $\quad$ sugerir nuevos modelos de solución de problemas;

- proveer nuevas definiciones conceptuales que puedan ayudar a otros científicos a resolver problemas;

- $\quad$ superar criterios de sus antecesores, o bien, despertar nuevos criterios ya abandonados;

- ser capaz de explicar todos los datos que se reunieron bajo teorías relevantes;

- $\quad$ ser consistente con todas las teorías adicionales preexistentes con validez científica;

- $\quad$ guiarse por leyes naturales y explicarse en base a leyes naturales;

- $\quad$ ser contrastable con el mundo empírico; y,

- asumir que sus conclusiones son provisionales para siempre.

Mantenía Lakatos (1975) que, mientras una teoría científica tenga algo a su favor, no conviene eliminarla hasta que se posea una teoría mejor; entiende que deba dársele un tiempo para que se modifique, pudiéndose enfrentar mejor a las anomalías que le afecten. 


\section{Hacia UnA TeOría SOBRE EL RENDIMIENTO ACADÉMICO}

No es objetivo del presente trabajo entrar en la revisión y análisis conceptual sobre rendimiento académico, ni siquiera de manera superficial. No existe investigador alguno sobre esta cuestión que no comience su discusión poniendo de relieve, y de antemano, la gran dificultad que ello conlleva, incluso, a modo de excusa previa, tratando de huir de posibles conceptos aminorados que lleven una desconsideración académica o científica sobre la amplitud e interés de este ámbito pedagógico, social y económico que abarca por igual a toda la sociedad. No olvidemos, a modo de ejemplo y reflexión general, que los problemas educativos, con sus resultados y consecuencias, son una constante en las preocupaciones individuales y colectivas del ser humano, siendo infrecuente la familia que no condiciona su horario semanal y los períodos de vacaciones al ritmo escolar, dado el alto porcentaje de personas que siguen enseñanzas públicas o privadas, formales o informales, y todo ello condicionado, también, y de forma muy importante, a veces, decisiva, en función del rendimiento académico obtenido en términos de éxito o fracaso.

El tema del rendimiento académico se ha visto reflejado constantemente en el ámbito de la investigación pedagógica durante los últimos treinta años en nuestro país, tanto en las convocatorias y proyectos públicos de investigación, como en la investigación llevada a cabo en los diferentes departamentos universitarios. En un estudio bibliométrico sobre producción de trabajos sobre esta temática realizado por nosotros (Nieto y Pérez Serrano, 1992), recopilamos datos del período 19701990, que ascendían a 654 trabajos, si bien, muchos de ellos eran Tesinas que se realizaban bajo diseños de investigación basados en la correlación entre alguna variable de relieve (inteligencia y personalidad, fundamentalmente) y las calificaciones de los alumnos, o bien, trabajos, por propia iniciativa de los investigadores, basados en la aplicación de algún cuestionario y su correlación entre los datos obtenidos, sin olvidar, obviamente, las Tesis Doctorales realizadas, siendo de gran interés la mayoría de ellas. Se producía un progresivo avance en la investigación por equipos, esencialmente a partir de las convocatorias realizadas por el MEC a través de su correspondiente organismo de investigación.

Por lo tanto, entendemos que la importancia y trascendencia del tema siempre presenta rasgos de permanente actualidad sobre los que se requiere una investigación rigurosa y coordinada mediante una intencionada planificación administrativa que responda puntualmente a aquellos aspectos que presentan registros de mayor oscuridad, pero, sobre todo, orientados a generar una teoría que ayude a consolidar científicamente, hasta donde sea posible, el conocimiento sobre el rendimiento académico. Nos cuesta entender que este decisivo ámbito de conocimiento científico no constituya, o no llegue a constituir, un importante núcleo de interés temático específico en el diseño y formación académica de todos cuantos tengan por objetivo dedicarse a la actividad profesional docente. Es difícil comprender que se pueda trabajar profesionalmente entre determinados problemas sin estudiarlos y conocerlos adecuadamente. 
HACIA UNA TEORÍA SOBRE EL RENDIMIENTO ACADÉMICO EN ENSEÑANZA PRIMARIA A PARTIR DE LA INVESTIGACIÓN EMPÍRICA: DATOS PRELIMINARES

La pregunta a la que deseamos responder en el presente artículo es si, a partir del trabajo de investigación llevado a cabo en nuestro país, podemos hablar de una teoría sobre el rendimiento académico, consolidación de tal conocimiento, en qué condiciones y cómo entender y valorar científicamente tales aportaciones.

\subsection{Educación, pedagogía y rendimiento académico}

La investigación sobre rendimiento académico supone, al menos, ofrecer un marco de referencia teórico-práctico al profesional de la educación, especialmente a los orientadores escolares. El tema tiene, en sí mismo, entidad suficiente como para constituir una preocupación para cada uno de los elementos que configuran el proceso educativo (alumnos, padres, profesores...); si bien el objetivo esencial de la investigación es poner de manifiesto conclusiones generales, principios, leyes..., sin embargo, el educador debe asumir las conclusiones con el objetivo de enmarcar e interpretar tales hallazgos con la finalidad única e irrepetible de la persona o personas que tiene ante sí. Convendríamos, pues, que la investigación sobre el rendimiento académico no constituye un fin en sí misma, sino como una estrategia más, incluso la más idónea, para fundamentar la actuación profesional del educador. De ahí el interés esencial que subyace en la recopilación y análisis que se realiza en el presente trabajo.

El rendimiento académico no sólo es utilizado por los profesionales de la educación, sino que llega a esgrimirse como bandera de la calidad o no de un determinado sistema educativo, e incluso, de una determinada orientación ideológica. Sin embargo, las variables que operan en el hecho de educarse son demasiado numerosas como para que, en su mayor parte, escapen del control de los propios educadores; aún así, el rendimiento educativo es la razón de ser de las instituciones escolares: y éste es un dato crucial para conocer, comprender e interpretar la vida en el aula y cada uno de los elementos personales que la constituyen.

\subsection{Reflexiones previas sobre la investigación pedagógica}

Resulta pertinente hacer dos reflexiones previas de interés; una, general, nuestra opinión sobre la investigación en la Universidad, y otra, más centrada en la investigación pedagógica en particular.

Hemos manifestado y razonado en algún otro trabajo (Nieto y Rodríguez Conde, 2007) que la investigación en la Universidad no responde a un criterio de planificación previa de la propia institución universitaria, y que la estructura, dinámica e inquietudes de los Departamentos promueven y se preocupan por la implicación en la resolución de cuestiones docentes, pero no así en el tema de la investigación. Se puede asegurar que la investigación en la Universidad es consecuencia y resultado del interés acumulativo individual o de grupos que genera la propia institución, que, en la mayoría de los casos, podrían desarrollar su trabajo 
al margen de la Academia. Además, la valoración administrativa de tal actividad resulta muy dispersa y está sembrada de demasiadas dudas, al margen de manifestar que, obviamente, el reconocimiento económico no se puede catalogar ni considerar precisamente de espectacular.

Y una opinión más particular es aquella que nos induce a pensar que la investigación pedagógica no está avalada por la profundidad en los temas o núcleos de interés que sucesivamente han ido apareciendo a lo largo del tiempo, sino que aparecen temas de actualidad, que, en forma de modas, incitan la realización de trabajos más actuales y de más fácil y reconocido eco entre investigadores. Así, por ejemplo, en el momento presente, parece que todo lo que no sea investigar sobre las TIC y los entornos virtuales daría la impresión de no estar en el mundo vanguardista y real de la investigación pedagógica; pero de igual manera nos podríamos referir a períodos inmediatamente anteriores con temas referidos a la evaluación institucional o evaluación de centros educativos... de tal manera que parecen abandonados temas de permanente interés y actualidad, si bien es cierto que en ningún momento se aleja la idea de una investigación vinculada al concepto de eficacia y eficiencia del sistema educativo.

\subsection{Precisiones actuales sobre los diseños de investigación educativa}

Parece intuirse y aceptarse que la tendencia actual sobre los diseños de investigación educativa debe estar marcada por el claro objetivo de aprender haciendo. Y es que, en el momento presente, se suelen plantear dos movimientos convergentes orientados a la tarea de incrementar y optimizar la producción de conocimiento utilizable para elevar el nivel de calidad de la práctica educativa: por una parte, la investigación educativa basada en el diseño y, por otra, la exigencia de un conocimiento basado en la evidencia empírica.

Teniendo en cuenta ambas consideraciones, se intenta planear ambientes educativos innovadores (Brown, 1992), que se presentan con la pretensión de apoyar argumentos construidos alrededor de los resultados de la intervención y la innovación activa, tratando de comprender los procesos de enseñanza y aprendizaje en los que el propio investigador se halla inmerso. La investigación se centra en diseñar innovaciones y en explorar todos los aspectos integrados en la misma durante su puesta en operación.

Así pues, emulando al National Research Council, y en base al planteamiento realizado, los principios que de forma generalizada están sirviendo, y deberían servir, de guía para financiar la actividad investigadora (De la Orden, 2004, 26) giran en torno a los siguientes:

- plantear cuestiones significativas y relevantes que puedan ser investigadas de forma empírica;

- ligar investigación a teoría relevante;

- proporcionar una cadena de razonamiento manifiesta y coherente; 
HACIA UNA TEORÍA SOBRE EL RENDIMIENTO ACADÉMICO EN ENSEÑANZA PRIMARIA A PARTIR DE LA INVESTIGACIÓN EMPÍRICA: DATOS PRELIMINARES

- distinguir métodos y datos para facilitar y estimular el escrutinio y crítica del trabajo;

- intentar resultados que puedan replicarse y generalizarse.

De todos es sabido el alto grado de disfuncionalidad de los resultados y usos de la investigación educativa; el hecho de que la investigación pedagógica se justifique por el efecto optimizante del proceso intencional que supone educar no admite negar la imperiosa necesidad de llevar a cabo una investigación pedagógica orientada a la generación de sólidas teorías que puedan ser contrastadas empiricamente, dando con ello cumplida respuesta a la creación de un necesario conocimiento científico en torno a cuestiones pedagógicas de relevancia e interés.

\section{Temática Que aglutina la inVestigación Pedagógica en el PERÍOdo 1971-1998}

En la actualidad, existe una gran dispersión en la diversidad temática sobre la investigación pedagógica institucional en nuestro país; las competencias educativas de cada Comunidad Autónoma dificultan un estudio pormenorizado sobre qué y cómo se investiga en el ámbito educativo, si bien, los actuales medios tecnológicos de que disponemos atenúan tal dificultad. Es cierta la existencia de una interesante variabilidad temática y metodológica; pero también lo es el hecho de que, ante tal situación, se tienden a difuminar las responsabilidades de control de calidad.

A tenor de los datos de que disponemos, la investigación educativa se ha llevado a cabo bajo criterios de selección temática variada, si bien hemos de manifestar que bajo un control de escaso rigor metodológico, con resultados, a veces, excesivamente obvios y simplistas.

En el período 1985-1998, en la normativa de las correspondientes convocatorias se establecen temas prioritarios a subvencionar, que, por su interés, exponemos a continuación por orden cronológico:

TABLA 1. RELACIÓN CRONOLÓGICA DE TEMAS PRIORITARIOS A INVESTIGAR

\begin{tabular}{|c|c|}
\hline AÑO & TEMAS PRIORITARIOS \\
\hline 1985 & $\begin{array}{l}\text { - Planteamiento, seguimiento y evaluación de la reforma en las EEMM } \\
\text { - Evaluación de los usos y resultados de las nuevas tecnologías educativas } \\
\text { - Educación Especial } \\
\text { - Educación Compensatoria }\end{array}$ \\
\hline 1986 & $\begin{array}{l}\text { - Oferta educativa } \\
\text { - Organización, costes, financiación y eficiencia del sistema de enseñanza post- } \\
\text { obligatoria no universitaria } \\
\text { - Procesos de transición de la escuela a la vida activa } \\
\text { - Demandas presentes y futuras del sistema productivo al sistema educativo }\end{array}$ \\
\hline
\end{tabular}


HACIA UNA TEORÍA SOBRE EL RENDIMIENTO ACADÉMICO EN ENSEÑANZA PRIMARIA A PARTIR DE LA INVESTIGACIÓN EMPÍRICA: DATOS PRELIMINARES

\begin{tabular}{|c|c|}
\hline 1988 & $\begin{array}{l}\text { - Disposiciones encaminadas a la reforma del sistema } \\
\text { - Actitudes y expectativas de los agentes educativos hacia los procesos de } \\
\text { reforma } \\
\text { - Interacción educativa } \\
\text { - Desarrollos curriculares potencialmente útiles para el sistema educativo como } \\
\text { un todo }\end{array}$ \\
\hline 1989 & $\begin{array}{l}\text { - Diseño curricular } \\
\text { - Evaluación de innovaciones metodológicas específicas } \\
\text { - Actitudes de alumnos y padres hacia los procesos de reforma } \\
\text { - Desarrollo y evaluación sistemática de nuevos materiales didácticos } \\
\text { - Educación de la mujer } \\
\text { - Últimos avances de la investigación sobre los efectos de los nuevos medios }\end{array}$ \\
\hline 1990 & - Materiales curriculares \\
\hline 1992 & $\begin{array}{l}\text { - El profesorado } \\
\text { - Igualdad de oportunidades }\end{array}$ \\
\hline 1993 & $\begin{array}{l}\text { - Imagen social y profesional de la enseñanza } \\
\text { - Evaluación de los aprendizajes }\end{array}$ \\
\hline 1994 & - Educación y televisión \\
\hline 1995 & $\begin{array}{l}\text { - Organización y funcionamiento de los centros docentes } \\
\text { - Resultados de la evaluación del aprendizaje de los alumnos y las decisiones de } \\
\text { promoción y titulación } \\
\text { - Atención a la diversidad y educación intercultural } \\
\text { - Desarrollo profesional de los docentes }\end{array}$ \\
\hline 1996 & $\begin{array}{l}\text { - Desarrollo de la LOGSE } \\
\text { - Participación, evaluación y gobierno de los centros docentes } \\
\text { - Compensación de desigualdades }\end{array}$ \\
\hline 1998 & $\begin{array}{l}\text { - Mejora de la calidad de la educación } \\
\text { - Compensación de desigualdades } \\
\text { - Educación y valores }\end{array}$ \\
\hline
\end{tabular}

Sin duda, en la relación anterior aparece reflejado todo un catálogo temático, a modo de núcleos de interés, del que no podemos negar su relación directa, de una u otra forma, en uno u otro nivel educativo, con el rendimiento académico. Sin embargo, en ningún caso aparece específicamente como tema el rendimiento académico, aunque en la clasificación posterior que realiza el CIDE (2002), y que abarca desde 1971, una vez ordenados los temas investigados en función de su número y porcentaje, comprobamos los siguientes datos: 
HACIA UNA TEORÍA SOBRE EL RENDIMIENTO ACADÉMICO EN ENSEÑANZA PRIMARIA A PARTIR DE LA INVESTIGACIÓN EMPÍRICA: DATOS PRELIMINARES

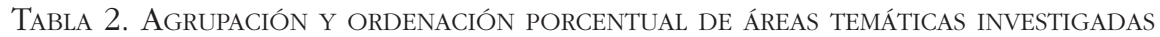

\begin{tabular}{|lrr|}
\hline Área temática & $\mathrm{N}$ & $\%$ \\
\hline Currículum & 126 & 13,7 \\
\hline Formación, actuación y situación del profesorado & 110 & 12,0 \\
\hline Procesos de enseñanza y aprendizaje & 109 & 11,9 \\
\hline Evaluación del rendimiento académico & 100 & 10,9 \\
\hline Igualdad de oportunidades en educación & 87 & 9,6 \\
\hline Sociología de la educación & 64 & 7,0 \\
\hline Desarrollo evolutivo y educación & 58 & 6,3 \\
\hline Educación y transición a la vida activa & 49 & 5,3 \\
\hline Política, reforma educativa y sistema educativo & 40 & 4,4 \\
\hline Evaluación del sistema educativo, de programas educativos & 39 & 4,2 \\
\hline y de centros escolares & 39 & 4,3 \\
\hline Tecnología de la educación & 30 & 3,3 \\
\hline Organización y dirección de centros escolares & 27 & 2,9 \\
\hline Pluralismo cultural y lingüístico en educación & 15 & 1,6 \\
\hline Economía de la educación & 13 & 1,4 \\
\hline Orientación educativa & 8 & 0,9 \\
\hline Teoría e Historia de la Educación & 3 & 0,3 \\
\hline Métodos y meta-análisis en investigación educativa & 917 & 100 \\
\hline TOTAL & & \\
\hline
\end{tabular}

Observamos que el rendimiento académico aparece vinculado a la evaluación y supone el 10\% del total de investigaciones subvencionadas, si bien, las clasificaciones de trabajos en otras áreas temáticas también convergen en la optimización de las instituciones y mejora del rendimiento de los alumnos. Siendo un tema importante, no parece tener atención continuada, ni responder a una programación constante que, en nuestra opinión, el tema merecería.

Por otra parte, accediendo a la base de datos Redinet hemos encontrado, bajo el descriptor rendimiento académico, la recopilación de 647 trabajos, aunque, en muchas ocasiones son simples referencias, con datos muy escasos, modestos resúmenes en forma de parcos y simples renglones descriptivos. Y ésta es una base de datos pública, de fácil acceso para cualquier ciudadano lector o investigador, que ha de servirnos de guía y referencia en el presente trabajo con el fin de llegar a razonar y formular las denominadas teorias de alcance intermedio sobre el rendimiento académico.

Vaya por delante el explícito reconocimiento de la dificultad que supone explicar el rendimiento académico, como por otra parte recogen las investigaciones más citadas en nuestro país sobre esta temática y a las cuales haremos cumplida y justa 
referencia en trabajos posteriores (Gimeno, 1976; Pelechano y otros, 1977; Carabaña, 1979; Pérez Serrano, 1981; Rodríguez Espinar, 1982; Tourón, 1984; Molina y García Pascual, 1984).

Para situarnos ante la dificultad comprensiva del tema, valga como reflexión previa la realizada por Gimeno (1976, 16), cuando manifestaba, después de una amplia investigación empírica, que "las aptitudes, actitudes, aspiraciones, intereses personales... del hombre vienen condicionados por las influencias de su medio ambiente", para continuar afirmando "que el entorno socio-cultural del alumno condiciona sus logros en la educación que imparten los establecimientos escolares", afirmando rotundamente que "la evidencia de esta influencia es hoy indudable", lo que nos sitúa ante una palpable y transparente realidad, cual es que el rendimiento académico es un producto condicionado por múltiples variables, factores y circunstancias a las que nos deberemos acercar a través de la investigación empírica, por mucha dificultad que ésta plantee en su diseño y estrategia metodológica.

\section{4. ¿ES POSIBLE UN META-ANÁLISIS?}

Alejándonos de la permanente actitud de fingir interpretaciones académicamente correctas, manifestamos con claridad que la posibilidad de realizar un metaanálisis con todo el volumen de información de que disponemos no es factible, o, al menos, no es posible realizarlo correctamente; quizás, en el mejor de los casos, sólo parcialmente y con determinadas investigaciones.

Aunque no es objetivo del presente trabajo la realización de un meta-análisis sobre investigaciones acerca del rendimiento académico, sino, tal y como hemos mencionado, preguntarnos sobre la posibilidad de generar una teoría en torno al tema, parece razonable hacer alguna mención puntual y de interés para disipar cualquier incorrecta interpretación metodológica sobre nuestra revisión y valoración posterior.

El meta-análisis es una técnica con grandes posibilidades de análisis conjunto y global que supone la integración estructurada y sistemática de la información obtenida en diferentes estudios, identificándolos y revisándolos de forma controlada con el objetivo de establecer una estimación cuantitativa sintética de todos ellos, con un poder estadístico superior a los estudios que incluye. Exige el cumplimiento de ciertos requisitos, el fundamental, por lógica, aunque no el único importante, es que las investigaciones presenten cierta homogeneidad, en especial, tanto en el diseño como en las técnicas de análisis, al margen de otros puntuales aspectos a tener en consideración con el fin de evitar importantes sesgos.

Y si algo hubiera que resaltar como reflexión constante es que, en ningún caso, el meta-análisis va a superar en calidad la propia calidad de los trabajos incluidos en el estudio; de ahí la condición previa que debe regir la selección de trabajos a incluir en el meta-análisis. 
HACIA UNA TEORÍA SOBRE EL RENDIMIENTO ACADÉMICO EN ENSEÑANZA PRIMARIA A PARTIR DE LA INVESTIGACIÓN EMPÍRICA: DATOS PRELIMINARES

La revisión bibliográfica que hemos realizado presenta y ofrece una gran y compleja diversidad, tanto en los fines, objetivos y selección de variables, como en el diseño, metodología empleada, técnicas de análisis... que, en lógica con las exigencias técnicas del meta-análisis, no permiten una integración e interpretación cuantitativa, aspectos que, como ya hemos manifestado, quedan fuera de discusión en el presente trabajo. Sin embargo, ello no es óbice para realizar alguna razonable y coherente sintesis de investigaciones sobre rendimiento académico apoyándonos en técnicas que se desarrollaron previamente a la aparición del metaanálisis, como fueron la revisión narrativa clásica y las revisiones sistemáticas. Por lo tanto, convenimos que meta-análisis, revisión sistemática y revisión narrativa clásica son términos para referirnos a distintas realidades, si bien, el meta-análisis, por lógica científica, incluye la revisión sistemática.

Uno de los principales sesgos que afectan a cualquier tipo de revisiones es el denominado sesgo de publicación. Resulta ya conocido (por evidente) a lo largo de la evolución científica que muchos trabajos de investigación, en su mayoría con resultados negativos, no suelen llegar a publicarse (entendiendo por negativos aquellos trabajos en cuyos resultados no se aprecian diferencias significativas o no están acorde con la previa formulación de hipótesis), sin olvidarnos del sesgo de selección, que compromete la actividad del propio investigador que realiza la revisión.

Nuestro planteamiento, que estimamos oportuno al fin deseado, pretende partir de la exposición descriptiva de resultados de una amplia selección de investigaciones recogidas en la base de datos Redinet, a que hacemos referencia, seleccionados en función de la antigüedad u orden de realización. La base de datos, en el momento de realizar la revisión, dispone de 648 trabajos. Dado que varios de ellos no se circunscriben a investigaciones propiamente dichas, sino a estudios o reflexiones teóricas sobre la cuestión, y algunos otros se centran en trabajos relacionados con alumnos de otros países, retiramos de la base de datos el $8,5 \%$ de los mismos, sometiendo a análisis el 91,5\% de los trabajos restantes, lo que supone 552 investigaciones a analizar. En la tabla siguiente establecemos una mera clasificación y descripción de frecuencias y porcentajes en función de las categorías establecidas en el sistema educativo.

En la tabla siguiente realizaremos una distribución de frecuencias en función del número de investigaciones llevadas a cabo y que constan en la base de datos mencionada, conforme a los criterios establecidos previamente y en la que añadimos, horizontalmente, el correspondiente cálculo porcentual. Podemos afirmar que, una vez revisada la información, la mayoría de las investigaciones llevadas a cabo se centran en la edad de 13-14 años, hecho que supone que en la tabla aparezca un elevado porcentaje de trabajos hasta 1990 en la categoría primaria, para trasladarse, a partir de esa fecha, progresivamente, hacia la categoría secundaria, como consecuencia de la reestructuración del sistema educativo desde esa fecha, dado que en nuestra clasificación hemos mantenido el criterio de estructura por categorías, en función de la establecida legalmente. Destacar el 20\% de media porcentual en la categoría universidad, si bien, hemos de manifestar que casi la mitad 
de ellas se centran en describir y analizar porcentajes de éxito o fracaso en función de las calificaciones obtenidas, así como la ausencia significativa de investigaciones entre niveles o categorías, además de una casi ausencia total de estudios longitudinales. Resaltar el bajo porcentaje de investigaciones experimentales, estando la mayoría basadas en trabajos empíricos descriptivos, correlacionales y, en menor medida, comparativo-causales.

TABla 3. Distribución de fRECUENCiAs de inVEstigaciones Sobre Rendimiento aCadémico. Período 1970-2007

\begin{tabular}{|c|c|c|c|c|c|c|c|c|c|c|c|c|}
\hline \multirow{2}{*}{$\begin{array}{l}\text { Períodos } \\
\text { temporales }\end{array}$} & \multicolumn{2}{|c|}{ Primaria } & \multicolumn{2}{|c|}{ Secundaria } & \multicolumn{2}{|c|}{ Univers. } & \multicolumn{2}{|c|}{ Prim/Secu. } & \multicolumn{2}{|c|}{ Secu/Univ. } & \multicolumn{2}{|c|}{ TOTAL } \\
\hline & $\mathrm{N}$ & $\%$ & $\mathrm{~N}$ & $\%$ & $\mathrm{~N}$ & $\%$ & $\mathrm{~N}$ & $\%$ & $\mathrm{~N}$ & $\%$ & $\mathrm{~N}$ & $\%$ \\
\hline 1970-1980 & 16 & 50,0 & 8 & 25,0 & 8 & 25,0 & 0 & 0,0 & 0 & 0,0 & 32 & 100,0 \\
\hline 1981-1985 & 53 & 56,0 & 14 & 15,0 & 19 & 20,0 & 8 & 8,4 & 1 & 1,1 & 95 & 100,0 \\
\hline 1986-1990 & 82 & 53,2 & 39 & 25,3 & 23 & 14,9 & 10 & 6,5 & 0 & 0,0 & 154 & 100,0 \\
\hline 1991-1995 & 45 & 48,4 & 21 & 22,6 & 18 & 19,4 & 6 & 6,5 & 3 & 3,2 & 93 & 100,0 \\
\hline $1996-2000$ & 19 & 24,1 & 37 & 46,8 & 19 & 24,1 & 4 & 5,1 & 0 & 0,0 & 79 & 100,0 \\
\hline 2001-2007 & 25 & 25,3 & 45 & 49,5 & 24 & 24,2 & 4 & 4,0 & 1 & 1,0 & 99 & 100,0 \\
\hline TOTAL & 240 & 43,5 & 164 & 29,6 & 111 & 20,1 & 32 & 5,8 & 5 & 1,0 & 552 & 100,0 \\
\hline
\end{tabular}

5. DESCRIPCiÓN DE FACTORES DETERMINANTES O INFLUYENTES EN EL RENDIMIENTO ACADÉMICO EN ENSEÑANZA PRIMARIA

Una correcta selección sistemática constituye una herramienta excelente para encontrar evidencias sobre el tema objeto de análisis, máxime teniendo presente el objetivo al que se desea llegar. Para evitar cierta confusión interpretativa, establecemos como criterio seleccionar los resultados más nítidamente reflejados como conclusiones en las investigaciones llevadas a cabo en el nivel de enseñanza primaria. Como podemos observar, es en la década de los 80 donde se produce un evidente despegue de la investigación en el ámbito temático del rendimiento académico. Seleccionamos todas las investigaciones correspondientes a cada uno de los cinco bloques cronológicos que exponemos a continuación, mostrando descriptivamente las conclusiones esenciales y que presenten un adecuado grado de claridad comprensiva.

Si la primera referencia bibliográfica de que disponemos en nuestro país sobre predicción del rendimiento académico es el trabajo de Secadas (1952), y ya en los años 60, fundamentalmente, los de García Yagüe y López Aberártury (1964), es a partir de la década de los 70 cuando comienza a producirse un progresivo interés por este ámbito temático, en cuyo período, hasta 1980, hemos recopilado 32 investigaciones de la base de datos Redinet, de las cuales, el 50\% corresponden al nivel 
HACIA UNA TEORÍA SOBRE EL RENDIMIENTO ACADÉMICO EN ENSEÑANZA PRIMARIA A PARTIR DE LA INVESTIGACIÓN EMPÍRICA: DATOS PRELIMINARES

primario de la enseñanza. Es en este período de años en el que situamos nuestra inicial descripción de conclusiones.

TABla 4. Conclusiones esenciales: PERÍOdo 1970-1980

a) El nivel social de las familias predice la inteligencia verbal de los sujetos

b) La comprensión verbal favorece el rendimiento académico de los alumnos

c) El autoconcepto aparece como un factor predictivo esencial para explicar el rendimiento

d) La preescolarización actúa como una variable definitoria del rendimiento académico, sobre todo en el primer curso de primaria, siendo más acusada tal diferencia en el área de lenguaje

e) Residir en hábitat urbano favorece una preparación cognoscitiva adecuada a las exigencias del trabajo escolar

f) Los alumnos procedentes de sectores sociales más desfavorecidos obtienen peores resultados académicos

g) Los deficientes mentales no sufren un especial rechazo en su interacción con alumnado sin discapacidad; el sociograma muestra que pueden ser bien acogidos por sus compañeros

h) La inteligencia presenta una estrecha relación con el rendimiento académico

En este período ya aparecen una serie de conclusiones esenciales que se van a convertir en una constante a lo largo de los años como son el autoconcepto, el origen social, la comprensión verbal, la capacidad intelectual y el preescolar como elementos determinantes del rendimiento.

El resumen de la tabla siguiente correspondiente al período cronológico que se menciona nos muestra alguna obviedad, alguna dudosa conclusión y de escaso interés, y, especialmente, dos núcleos fundamentales de interés que enlazan con valoraciones ya realizadas anteriormente, como son la importancia del autoconcepto y el origen social del alumno como elementos determinantes del rendimiento académico.

\section{TABla 5. Conclusiones eSENCIALES: PeríOdo 1981-1985}

a) La introducción de metodologías experimentales en el área de ciencias aumenta la satisfacción de los alumnos, la responsabilidad y el rendimiento académico

b) El fracaso escolar se produce en niños con bajo coeficiente intelectual y desajuste emocional

c) La alteración de la relación maestro-alumno puede ser causa y/o efecto de desajuste emocional

d) El rendimiento académico de los alumnos, al final de la EGB, no alcanza, en gran medida, la madurez académica establecida

e) No existen diferencias significativas de rendimiento global respecto a la variable sexo 
f) Los alumnos con menor rendimiento académico proceden de grupos familiares con menor nivel económico y más bajo nivel de estudios de su familia

g) El autoconcepto relaciona positivamente con el rendimiento académico

h) El rendimiento académico está relacionado, fundamentalmente, con factores de índole social, más que con factores intrínsecos al propio sujeto

i) En el ciclo inicial de EGB, las pruebas que miden factores cognoscitivos tienen mayor validez predictora que las de rendimiento; en esta edad, las pruebas de personalidad tienen un valor predictivo nulo

j) Al finalizar la EGB, los niños no alcanzan el nivel óptimo de desarrollo en el lenguaje verbal, así como, tampoco, en lectoescritura

k) El rendimiento está mediatizado por el origen y situación profesional de la familia

1) Los alumnos superdotados proceden, de forma predominante, de familias con estudios medios o superiores, de colegios privados y pocos hermanos

m) A mayor nivel socioeconómico y de estudios en las familias, mayor es el éxito académico en los alumnos del ciclo medio

n) Los elementos de intervención positivos inciden favorablemente en el rendimiento de los alumnos de ciclo medio

o) La mayor capacidad de memoria visual, percepción visual y capacidades visocontructivas relacionan favorablemente con el éxito escolar

p) Las dinámicas de educación en valores se relacionan con actitudes positivas hacia uno mismo como factor de maduración personal

q) Los factores que parecen incidir favorablemente en un buen rendimiento académico son las expectativas de los padres, la participación de éstos en la tareas escolares y el nivel cultural y socioeconómico de la familia

r) Los factores extraescolares tienen un gran peso en el rendimiento académico

s) Las aptitudes verbales maduran con mayor rapidez en el sexo femenino que en el masculino.

En la tabla siguiente exponemos una larga relación de conclusiones, y que pertenecen, creemos, al período de mayor inquietud e interés por investigar sobre el rendimiento académico, que, no obstante, es en el que se llevan a cabo mayor número de investigaciones en este nivel académico que estamos analizando.

\section{TABla 6. Conclusiones esenciales: PERÍODO 1986-1990}

a) El alumno repetidor suele ser ignorado por sus compañeros; la variable que marca las relaciones dentro de la clase es el rendimiento académico. El alumno repetidor percibe que el profesor trata de forma diferente a unos niños que a otros, condicionado por el rendimiento académico de cada uno de ellos

b) Los niños con deficiencias psíquicas y carencias familiares son capaces de entusiasmarse y trabajar en proyectos comunes en el aula 
HACIA UNA TEORÍA SOBRE EL RENDIMIENTO ACADÉMICO EN ENSEÑANZA PRIMARIA A PARTIR DE LA INVESTIGACIÓN EMPÍRICA: DATOS PRELIMINARES

c) Tras dos años de escolarización, los escolares aún no han adquirido el deseable conocimiento lingüístico formal, dificultando el uso del lenguaje narrativo

d) El aprendizaje se asienta en relaciones sociales y la integración socioafectiva de los alumnos

e) La repetición de curso no es eficaz en el 50\% de los alumnos que no consigue el graduado escolar en final de la EGB

f) Los rendimientos específicos discriminan secundariamente entre repetidores y no repetidores

g) La motivación discrimina significativamente entre repetidores y no repetidores

h) Las diferencias encontradas en técnicas básicas entre repetidores y no repetidores no son excesivamente elevadas

i) La asistencia al preescolar, el tipo de centro y la clase social producen diferencias significativas con el rendimiento académico medido a través de pruebas específicas, si bien, tales diferencias no son tan acusadas teniendo en cuenta las calificaciones escolares

j) Existe interdependencia entre actitudes sociales positivas, reacciones emocionales y rendimiento académico

k) Las reacciones emocionales tienen una mayor incidencia en el rendimiento que las variables actitudinales

1) Las mayores expectativas al terminar los estudios inciden favorablemente en un mayor rendimiento académico, tanto medido con pruebas específicas como con las calificaciones escolares

m) El antiautoritarismo incide favorablemente en el rendimiento, siendo menor el rendimiento de los autoritarios

n) Los mejores predictores aptitudinales del rendimiento son el verbal, seguido del numérico y del razonamiento, tanto medido mediante pruebas específicas como con las calificaciones, si bien es superior cuando se utilizan las pruebas específicas

o) El estatus socioeconómico tiene una escasa incidencia en la relación aptitud-rendimiento

p) La variable sexo muestra una débil influencia en el rendimiento global; no obstante, sí existen diferencias significativas a favor de los niños en matemáticas, y a favor de las niñas, en conocimientos lingüísticos

q) La aptitud espacial y mecánica incide negativamente en los conocimientos lingüísticos, lo que parece estar relacionado con el sexo

r) La preescolarización y preparación cognoscitiva que posee el alumno en primero de primaria resulta determinante en el rendimiento académico posterior

s) El rendimiento difiere según el nivel de estudios de los padres, no así en función de las variables sexo y tipo de centro

t) Las reacciones emocionales determinan mejor el rendimiento académico que las variables actitudinales

u) La actitud que más discrimina favorablemente el rendimiento académico es el antiautoritarismo; también, a mayores expectativas, mayor rendimiento

v) El hecho de repetir curso afecta negativamente el nivel de autoconcepto académico 
x) El autoconcepto correlaciona positivamente y de forma elevada con el rendimiento académico; así mismo, se comprueba que el autoconcepto puede ser mejorado con actividades programadas a tal fin, fundamentalmente por la vía de la motivación

y) El rendimiento previo revela mayor capacidad predictiva del rendimiento posterior, siendo la inteligencia general verbal, la aptitud numérica y la comprensión verbal las que se muestran como segundos predictores

z) Ni los intereses vocacionales ni los rasgos de personalidad presentan valor predictivo de interés

aa) La inteligencia es un determinante del rendimiento en los primeros cursos de primaria, siendo el autoconcepto académico un factor esencial del rendimiento, y no así la personalidad

ab) Las variables de tipo pedagógico discriminan de forma significativa más que ninguna otra

ac) El perfil psicopedagógico de los alumnos con bajo rendimiento se caracteriza por su menor capacidad para adquirir los conocimientos impartidos por el centro escolar, mayor reacción emocional de ansiedad, mayor hostilidad en sus relaciones sociales, peor autoconcepto de sí mismos como persona y como estudiante y menores aspiraciones profesionales

ad) Los alumnos con elevado nivel de comprensión y velocidad lectora muestran un brillante expediente académico

ae) Se detecta una manifiesta incapacidad para aplicar o transferir conceptos teóricos a situaciones prácticas

af) El mejor predictor del rendimiento son las aptitudes; el nivel cultural de la familia tiene una relación causal con las aptitudes

ag) La velocidad lectora y los hábitos de estudio inciden favorablemente en el rendimiento académico

ah) Las reacciones emocionales determinan mejor el rendimiento que las variables actitudinales; y a mayores expectativas al finalizar los estudios, mejor rendimiento

ai) El autoconcepto, como variable que incide en el rendimiento, se muestra estable y resistente al cambio; se requiere una intervención positiva, sistemática y duradera de personas significativas para el alumno

Sin perder la óptica de las conclusiones referidas al período anterior, en éste se vuelve a repetir la importancia del autoconcepto como determinante del rendimiento, siguiendo con temas de gran interés, como son la importancia del preescolar, cómo afecta la repetición de curso, la importancia de las expectativas, la motivación, los factores emocionales, el antiautoritarismo, y, como en el período anterior, de una u otra forma, referencias al origen social y a la capacidad predictiva de variables relacionadas con el uso y dominio lingüistico. En cualquier caso, no reproducen, y así hay que manifestarlo, réplicas intencionadas a conclusiones obtenidas en otras investigaciones, donde los temas, las muestras, el diseño, la estrategia, los instrumentos de recogida de información y las técnicas de análisis llevadas a cabo deberían empezar a formar parte de toda una teoría sobre rendimiento académico que pretendemos captar y dar a conocer. 
HACIA UNA TEORÍA SOBRE EL RENDIMIENTO ACADÉMICO EN ENSEÑANZA PRIMARIA

A PARTIR DE LA INVESTIGACIÓN EMPÍRICA: DATOS PRELIMINARES

TABla 7. Conclusiones esenciales: Período 1991-1995

a) Los niños situados en un rango limítrofe intelectual mejoran las ejecuciones intelectuales tras una intervención de entrenamiento cognitivo

b) El nivel intelectual global y las competencias lingüísticas en primero de primaria predicen positivamente el rendimiento académico

c) Los alumnos de aulas de educación especial con deficiencia mental ligera y límites deberían estar integrados en aulas ordinarias; estos alumnos obtienen un rendimiento similar a otros alumnos con dificultades de aprendizaje que tienen una escolarización normal, mientras que si permanecen en el aula de educación especial consiguen rendimientos más bajos en pruebas estandarizadas., siendo los más jóvenes los que obtienen mejores resultados

d) Las niñas están mejor adaptadas que los niños en relación a sus compañeros, profesores y la escuela como comunidad

e) Los alumnos procedentes de niveles socioeconómicos bajos o muy bajos presentan problemas de adaptación a la comunidad educativa, si bien el nivel cultural de las familias no influye en la adaptación escolar

f) El nivel socioeconómico determina el tiempo de la duración de la escolaridad, factor decisivo en la aparición de la inadaptación escolar

g) No se produce relación significativa entre autoestima y deprivación

h) Existen diferencias significativas en el rendimiento a favor de los varones en el área de Ciencias

i) Existen diferencias significativas en el rendimiento a favor de las mujeres en el área de Lengua

j) No existen diferencias significativas en función del sexo en matemáticas ni en rendimiento total

k) Los monolingües obtienen mejores resultados que los bilingües en variables lingüísticas

1) Las variables predictoras del rendimiento global presentan diferente valor de predicción en alumnos bilingües y monolingües

A todo lo anteriormente comentado añadimos la importancia de la competencia lingüistica como elemento destacado de investigación, al que se añaden los temas de integración escolar y bilingüismo. Vuelve a incidirse en variables socioeconómicas como factores que discriminan el rendimiento y la adaptación escolar, así como la importancia de la variable sexo en algunas cuestiones puntuales, manifiesta en unos casos, dudosa en otros, pero presente en las investigaciones casi siempre.

A todas las referencias anteriores, añadimos un nuevo período de conclusiones tal y como hemos establecido. Así, 
SANTIAGO NIETO MARTÍN

HACIA UNA TEORÍA SOBRE EL RENDIMIENTO ACADÉMICO EN ENSEÑANZA PRIMARIA

A PARTIR DE LA INVESTIGACIÓN EMPÍRICA: DATOS PRELIMINARES

TABla 8. Conclusiones esenciales: Período 1996-2000

a) Los trece estilos intelectuales definidos por Sternberg muestran una contribución independiente y significativa en la explicación del rendimiento académico

b) Los reiterados fracasos escolares predisponen la aparición de estilos cognitivos depresógenos

c) El autoconcepto configura el rendimiento académico, al igual que el contexto familiar y las expectativas familiares

d) Los alumnos mejoran significativamente la rapidez y la comprensión lectora, así como la disminución del número de errores, al leer en voz alta

e) El programa de Apoyo y Desarrollo de la Inteligencia obtiene una mejora significativa en las áreas curriculares fundamentales, además de elevar la motivación de los niños

f) Los programas para mejorar el rendimiento escolar deben centrarse en el desarrollo de los receptores sensoriales como la visión, audición y tacto, así como en programas neuromotores

g) El porcentaje de fracaso escolar es similar entre los superdotados y el resto de alumnos considerados normales

h) La atención a las necesidades educativas especiales de los alumnos con altas habilidades ha de llevarse a cabo, primordialmente, dentro del aula ordinaria y del ámbito normal de la clase

Vuelve a surgir el autoconcepto como elemento de interés, la importancia de la competencia lectora, la mejora de receptores sensoriales y neuromotores como determinantes del rendimiento, y, especialmente, la necesidad de implantar programas de Apoyo y Desarrollo de la Inteligencia.

Como se puede fácilmente comprobar, se amplía el listado de temas de investigación, con algunos aspectos repetitivos y difusos. No obstante, se observa en ésta y otras tablas anteriores, una paulatina aparición de conclusiones consecuencia de intervenciones pedagógicas. Veamos, pues, la última tabla de resultados:

TABla 9. CONClusiones ESENCIALES: PERÍODO 2001-2006

a) Mejores calificaciones en los centros de jornada partida, mientras que el profesorado de los centros de jornada continua manifiesta que este último modelo favorece el resultado de los alumnos

b) La participación en un Programa de Desarrollo Integral favorece el autoconcepto, inteligencia general, estrategias de aprendizaje, personalidad y rendimiento académico. El autoconcepto se configura como un elemento central de la personalidad y el mayor factor motivacional de la conducta del sujeto, esencialmente en mejoras no académicas

c) Las diferentes agrupaciones musicales en los colegios (danza, conjuntos vocales e instrumentales) favorecen la autoestima, la atención y percepción, la tolerancia, un comportamiento más tolerante hacia sí mismo y hacia los demás 
d) Los efectos de la cooperación grupal se muestran positivos tanto en la interacción educativa del grupo como en el rendimiento académico general

e) El trabajo cooperativo en el aula mejora el rendimiento académico

f) Los hijos que perciben a los padres como democráticos y permisivos obtienen puntuaciones académicas más elevadas

g) Las expectativas de control de los resultados académicos futuros son el mejor predictor del rendimiento

h) El entrenamiento en la elaboración de resúmenes favorece la mejora de habilidades metacomprensivas de la lectura, de la comprensión lectora y el rendimiento académico

De la tabla anterior extraemos, como temas novedosos, la investigación sobre la jornada continuada o partida, el interés por la música y la cooperación grupal y trabajo cooperativo como factor coadyuvante del rendimiento académico, apareciendo como elemento más repetitivo, nuevamente, el autoconcepto y, en menor medida, las expectativas, el antiautoritarismo, Programas de Desarrollo Integral, formación y maduración lingüística...

En la mayoría de las investigaciones no aparece claramente diferenciada la medición del rendimiento, normalmente medido mediante calificaciones del profesor o bien pruebas específicas. En cualquier caso, aparecen reflejadas muchas tendencias y pocos teoremas, sin aval metodológico suficiente; incluso se producen conclusiones generales, simplemente, por aproximación a las hipótesis que se formulan. Por supuesto, no existe investigación alguna que haga referencia al tamaño del efecto, que, con los programas informáticos actuales, bien debería tenerse en cuenta.

En muchos de los trabajos analizados no se observa claridad alguna en la estrategia metodológica, con muestras que, a veces, resultan llamativas por su baja magnitud, en ocasiones, con modelos simplistas, lo que no evita, sin embargo, valorar la información proporcionada en sus justos términos y de la que pretendemos dar cuenta descriptiva en el presente trabajo a modo de estudio preliminar sobre la cuestión. En cualquier caso, sí parece demostrarse la insuficiencia de estudios, la ausencia de réplicas investigadoras, la falta de investigación sobre temas nucleares que van apareciendo como cruciales, así como comparaciones entre resultados obtenidos mediante diferentes instrumentos de medida.

Pero si alguna cuestión resalta llamativa es que prácticamente todos los estudios se llevan a cabo con muestras no probabilísticas accidentales o a propósito y un tamaño de ocasión o de circunstancias, sin ninguna referencia al error muestral, y con dudosa claridad cuando del nivel de confianza se trata. Y ésta es una situación que genera la lógica desconfianza al estar vinculada con la validez del proceso estadístico, y, en consecuencia, con la generalización de resultados. En ausencia de aleatoriedad en la elección de las muestras, existen estrategias de selección que en muy pocos estudios se llevan a cabo, como son, por ejemplo, muestras estratificadas o muestras por agrupamientos (cluster sampling). Como 
consolación, podemos refugiarnos en la idea que ya manejó Cattell hace cuarenta años, cuando afirmaba que al investigador en educación le resultaba prácticamente imposible seguir las recomendaciones del método aleatorio. Sin duda, la gran cantidad de investigaciones revisadas por nosotros en este trabajo vendrían a avalar la dificultad mostrada. Y aun estando totalmente de acuerdo con Rodríguez Espinar (1982, 66), cuando manifiesta que el principal objetivo de la investigación educativa radica en "la acción personal que puede ejercitarse sobre el sujeto en virtud de las directrices cualitativas que los datos nos aportan", no podemos obviar, cuando pretendemos la consecución de leyes y teorías científicas, el cumplimiento de ciertas exigencias científicas.

Por otra parte, si tenemos en cuenta la reflexión de Zikmund (2003, 144), al definir la encuesta como «un método de recogida de datos primarios mediante la comunicación con una muestra representativa de personas", nos daremos cuenta de que no se ha desarrollado por igual en algunas especialidades de las ciencias sociales, destacando su evolución, por ejemplo, en la Sociología o las Ciencias Políticas, y no así en Psicología o Pedagogía; mientras que en las primeras han alcanzado un alto grado de sofisticación, en las segundas recibe un tratamiento demasiado elemental, no llegando mucho más lejos de la mera recopilación de datos que propone la definición de partida, con escaso interés por el diseño apropiado, así como por el análisis e interpretación de los datos reunidos.

$\mathrm{Al}$ respecto, no podemos olvidar la dificultad para determinar relaciones causales entre variables, dada la imposibilidad de controlar el influjo de variables contaminantes cuando se trabaja en un contexto natural, así como, tampoco, mantener condiciones constantes, llevándose a cabo el trabajo investigador sobre una realidad estática, correspondiendo a un momento, a un lugar determinado y sobre un conjunto específico de sujetos. Los estudios longitudinales, como forma de paliar algunos inconvenientes, no suelen formar parte del elenco de investigaciones educativas que se llevan a cabo en nuestro país.

No podemos olvidar alguna referencia a los modelos explicativos del rendimiento escolar; aunque han sido elaborados a partir de los conocimientos proporcionados por la investigación, se constituyen en referentes esenciales para articular de forma coherente la planificación investigadora. A mediados del siglo pasado, Vernon (1952) elaboró aquello que podríamos considerar como un modelo preliminar al incluir un espectro amplio de factores a la hora de estudiar el rendimiento académico; desde entonces, se han ido perfilando modelos psicológicos, sociológicos, psicosociales, eclécticos... estableciendo predicciones del rendimiento desde las distintas variables del ámbito personal, familiar, escolar, comportamental... De gran interés resulta la recopilación y análisis que sobre este tipo de modelos realiza Antoni Adell (2006, 28), y lo que es más importante, las estrategias educativas que este mismo autor establece por cada uno de los ámbitos referidos. 
HACIA UNA TEORÍA SOBRE EL RENDIMIENTO ACADÉMICO EN ENSEÑANZA PRIMARIA A PARTIR DE LA INVESTIGACIÓN EMPÍRICA: DATOS PRELIMINARES

\section{HaCia El DESARROLlO DE TEORÍAS DE ALCANCE INTERMEDIO}

Procede preguntarnos, pues, si podemos aspirar a entenderlos dentro de una teoría general sobre el rendimiento académico, lo suficientemente amplia como para agrupar las grandes cantidades de detalles que se observan en las personas y en los diferentes colectivos educativos. No parece posible a la luz que irradia el volumen general, en cantidad y calidad, de investigaciones llevadas a cabo, lo que pone de manifiesto que, en general, la investigación pedagógica responde más a los intereses y necesidades puntuales de los investigadores que a una línea de trabajo coherente y consolidado que podría y debería establecerse previamente.

Entendemos, pues, que deberíamos retomar en nuestro estudio y análisis las denominadas teorías de alcance intermedio, tan debatidas en algunos ámbitos de las ciencias sociales, y plantearlas como necesidad de estudio y análisis acerca del rendimiento académico, en vez de entender este ámbito temático como un sistema teórico completo. Ello exige a los investigadores un compromiso científico diferente y asumir con humildad los cambiantes contextos intelectuales en que nos movemos, tratando de desarrollar teorías puntuales y especiales que guíen la investigación hacia problemas específicos dentro de un marco provisional y, siempre, en evolución. Se debería suponer que este ámbito temático avanzaría más en la medida en que el interés principal esté en el desarrollo de teorías de alcance intermedio, a modo de peldaños pedagógicos intermedios, que aclaren dudas sobre temas de interés puntual.

En ese sentido, creemos que se debería partir de planes interrelacionados con dos claros objetivos y una finalidad común:

a) diseñar teorías especiales de las cuales derivar hipótesis que se puedan investigar empíricamente;

b) desarrollar un esquema conceptual progresivo más general que promueva la consolidación de grupos de teorías especiales, y

c) elaborar planes y estrategias orientadas a mejorar los diferentes ámbitos de incidencia en el rendimiento académico.

La revisión sobre investigaciones acerca del rendimiento académico produce la impresión de no orientarse a un esquema general efectivo, donde cada trabajo llevado a cabo trata de desarrollar un "sistema propio" de teorías, entre otras razones, porque no se enmarcan a partir de esquemas teóricos investigadores anteriores, generando una fragmentación no acorde con objetivos científicos claros. Entendemos que el objetivo primordial debería orientarse a la consecución gradual de teorías de alcance intermedio, convirtiéndose en casos especiales de formulaciones más generales.

Sin duda, disponemos de muchos conceptos, muchas opiniones, muchas aproximaciones, pero de pocas teorías confirmadas; aparecen demasiadas suposiciones subyacentes, y cualquier cambio de orientación siempre será positivo; de lo contrario podemos vernos abocados a aceptar la concepción acuñada por Tennyson, 
cuando afirmaba que "nuestros pequeños sistemas tienen su día; tienen su día y dejan de ser".

Entendemos que las conclusiones del trabajo investigador constituyen un recurso intelectual de primera magnitud, pero también la investigación llega a generar y alcanzar un conflicto entre profesionales que va más allá de la propia pedagogía: toda opinión genera una contraopinión que induce a la alineación en alguna de las diferentes partes en conflicto. Y en esa dialéctica, se establecen más categorías de defensa, que, propiamente, de búsqueda de la verdad. No obstante, no debemos abdicar de exigir y autoexigirnos diseños de investigación acordes con precisiones coherentes que orienten adecuadamente la consecución del conocimiento científico.

Los resultados observados inducen a pensar en una investigación orientada al descubrimiento de hechos o situaciones, en algunos casos, muy puntuales y meramente descriptivos. Hemos observado que, en general, el investigador está cada vez menos motivado por el estudio del trabajo del otro. Creemos arriesgado decir, pero necesario manifestar, que ante el reciente Informe Pisa diciembre-2007 no parece que seamos capaces de ofrecer respuestas de mayor convicción que las meramente interpretativas generadas por el ambiente o estructura normativa. Y ésa es una mera simplificación, por interesada y oscura ocultación de elementos o factores que bien podrían dimanar de la detallada observación del conocimiento proporcionado por la investigación empírica, por deficitaria que sea.

Retomamos, pues, nuestro inicial planteamiento al pensar que la investigación revisada no va más allá de generar teorías limitadas, de predecir tendencias y observaciones predominantes que podrían no ser ciertas, pero que poseen una presunta verdad. En cualquier caso, recurriendo al hecho cierto, pero fácil, al que solemos acudir con frecuencia manifestando carecer de suficiente investigación al respecto, podemos afirmar que, salvo puntuales excepciones, no se dan uniformidades regulares generales, leyes causales... sin las cuales es difícil establecer reglas de interés cognoscitivo que orienten el cómo y el porqué del rendimiento... con el fin de establecer estrategias o programas puntuales de intervención educativa sobre aquellos factores fluctuantes que pudieran estar al alcance de la ciencia pedagógica.

No podemos ser vulnerables a la mera especulación. De ahí que sea preferible asumir sin reservas la conveniencia o necesidad de asumir teorías de alcance intermedio como forma de interpretar y avanzar en el campo del rendimiento académico, dadas las propiedades o características de esta teoría:

a) Forman grupos limitados de suposiciones, de las que han de derivarse hipótesis específicas que deben ser confirmadas por investigaciones sólidamente fundamentadas.

b) Estas teorías, ni están, ni permanecen, ni las consideramos separadas, sino que forman parte en una red más amplia de teorías que han de ir consolidándose y orientando a un conocimiento científico mucho más general y amplio. 
HACIA UNA TEORÍA SOBRE EL RENDIMIENTO ACADÉMICO EN ENSEÑANZA PRIMARIA A PARTIR DE LA INVESTIGACIÓN EMPÍRICA: DATOS PRELIMINARES

c) Las teorías no deben ser meramente descriptivas, sino lo bastante abstractas como para tratar diferentes esferas de la conducta personal, psicológica y social de la persona.

d) Este tipo de teorías analiza, comprueba y compara problemas macro y micropsicológicos y sociológicos.

e) Una teoría general y final sobre el rendimiento académico estará constituida por sistemas rigurosos bien construidos de teorías intermedias que orienten una teoría unificada pedagógicamente.

f) Es posible que muchas teorías de alcance intermedio vayan concordando consigo mismas, lo que facilitará todo un entramado sistema de pensamiento pedagógico.

g) Estas teorías de alcance intermedio irán estando en consonancia con formulaciones teóricas previas que proporcionan ideas, tácticas, modelos y estrategias pedagógicas a implantar.

En definitiva, creemos en la necesidad de especificar nuestra ignorancia sobre la cuestión, al establecer un sistema que conlleve progresivamente el conocimiento de lo ausente; reconozcamos que aún debemos saber y conocer más, y sentar las bases de un progresivo y mayor conocimiento. No se trata de ofrecer soluciones a cada problema, sino de esclarecer los problemas a la luz que genera el conocimiento científico existente.

\section{BiBLIOGRAFÍA}

Antoni Adell, M. (2006) Estrategias para mejorar el rendimiento académico de los adolescentes (2. ${ }^{a}$ edición). Madrid, Pirámide.

BAKer, T. L. (1997) Doing social Research. New York, McGraw-Hill.

Botella, J. y Gambara, H. (2002) Qué es el meta-análisis. Madrid, Biblioteca Nueva.

Brown, H. I. (1983) La nueva filosofía de la ciencia. Madrid, Tecnos.

CARABAÑa, J. (1979) Origen social, inteligencia y rendimiento académico al final de la EGB, Temas de Investigación Educativa, I, 29-71.

Cide (2002) Treinta años de investigación educativa institucional en España. Madrid, MEC, CD-ROM.

De la Orden, A. (2004) Producción, transparencia y uso del conocimiento pedagógico, en BuEndíA, L. y otros. Temas fundamentales en la investigación educativa. Madrid, La Muralla.

Echeverría, J. (1995) Filosofía de la ciencia. Madrid, Akal.

Escohotado Espinosa, A. (2006) Filosofía y metodología de las ciencias sociales. Madrid, Ediciones Académicas.

García Yagüe, J. y López Aberasturi, N. (1964) El pronóstico para los estudios del bachillerato elemental al nivel de ingreso, Revista de Psicología General y Aplicada, XIX (73), 523-526.

Gimeno SACRistán, J. (1976) Autoconcepto, sociabilidad y rendimiento escolar. Madrid, INCIE-MEC. 
SANTIAGO NIETO MARTÍN

HACIA UNA TEORÍA SOBRE EL RENDIMIENTO ACADÉMICO EN ENSEÑANZA PRIMARIA

A PARTIR DE LA INVESTIGACIÓN EMPÍRICA: DATOS PRELIMINARES

Gómez Rodríguez, A. (2005) Filosofía y metodología de las ciencias sociales. Madrid, Alianza Editorial.

Hawking, S. (2000) Breve historia del tiempo. México, Planeta Mexicana.

Hempel, C. G. (1995) Filosofía de la ciencia natural. Madrid, Alianza Editorial.

Holus, M. (1998) Filosofía de las ciencias sociales. Barcelona, Ariel.

KerLinger, F. (1985) Investigación del comportamiento. México, Interamericana.

Lakatos, I. (1975) The Methology of Scientific Research Programes. Cambridge, University Press.

- (1986) Pruebas y refutaciones. Madrid, Alianza.

Loscos, M. ${ }^{a}$ P. (1985) Meta-análisis sobre predicción del rendimiento escolar, Revista de Investigación Educativa, 3 (6), 221-235.

Mateo, J. (1985) Meta-análisis correlacional sobre estudios de rendimiento escolar en España, Revista de Investigación Educativa, 3 (6), 236-251.

Merton, R. (1980) Teoría y estructura social. México, Fondo de Cultura Económico.

Molina García, S. y Gracia Pascual, E. (1984) El éxito y el fracaso escolar en EGB. Barcelona, Laia.

Nieto, S. y Pérez Serrano, G. (1994) Estudios e investigaciones sobre rendimiento académico (1970-1990): análisis estadístico y bibliométrico, Revista Española de Pedagogía, 199 (3), 501-527.

Nieto, S. y Rodríguez Conde, M. ${ }^{a}$ J. (2007) Reconocimiento administrativo de la actividad investigadora en la Universidad. Reflexión acerca de los denominados sexenios de investigación a partir de unos datos empíricos, Enseñanza, 24, 315-346.

Pelechano, V. y otros (1977) Personalidad, inteligencia, motivación y rendimiento académico en BUP. La Laguna, ICE Universidad.

Pérez Serrano, G. (1981) Origen social y rendimiento escolar. Madrid, CIS.

Popper, K. (1978) La lógica del conocimiento científico. Madrid, Tecnos.

Rodríguez Espinar, S. (1982) Factores del rendimiento académico. Barcelona, Oikos-Tau.

SecadAs Marcos, F. (1952) Factores de personalidad y rendimiento escolar, Revista Española de Pedagogía, X (37), 77-87.

Touron, J. (1984) Factores del rendimiento académico en la Universidad. Pamplona, Eunsa. VARIOS (1976) Determinantes del rendimiento académico. Madrid, INCIE-MEC.

VERnON, P. E. (1952) La structure des aptitudes humaines. Paris, Presses Universitaires de France.

WARTOFsKy, M. W. (1987) Introducción a la filosofía de la ciencia. Madrid, Alianza Editorial. Yuren, M. ${ }^{a}$ T. (1978) Leyes, teorías y modelos. México, Trillas.

Zikmund, W. G. (2003) Fundamentos de investigación de mercados. Chile, Thomson. 